\title{
Suivi écologique de la dynamique des grands et moyens mammifères dans les clairières du parc national de Boumba Bek : cas du complexe de clairières de Pondo
}

\author{
IMBEY Moise Olivier4, $\dagger$, MBEZELE Junior Yannick NGABA ${ }^{1,3, \dagger}$, AHANDA Yanick Achille ${ }^{2,3}$, J.P. \\ BELINGA2 , G. ETOGA², Expedit FOUDA Bernard², Martin TCHAMBA ${ }^{3 *}$ \\ ${ }^{1}$ Collège de foresterie, Université d'Agriculture et de Foresterie de Fujian (FAFU), B.P. 350002, Fuzhou, Fujian, \\ China, \\ 2Fonds mondial pour la nature (WWF), B.P. 6776 Yaoundé, Cameroun, \\ 3Département de Foresterie, Faculté d'Agronomie et des Sciences Agricoles (FASA), Université de Dschang, B.P. \\ 222 Dschang, Cameroun. \\ ${ }^{4}$ Ecole des Eaux et Forêts de Mbalmayo, BP 69 \\ $\dagger$ Equal contribution \\ *Auteur correspondant, Tel : (237) 6777705412, E-mail : mtchamba@yahoo.fr
}

Original submitted on $10^{\text {th }}$ September 2019. Published online at www.m.elewa.org/journals/ on 31st December 2019 https://doi.org/10.35759/JABs.v144.4

\section{RESUME}

Objectifs : L'étude qui a été mené dans le parc national de Boumba Bek visait à connaitre le statut de la population animale de grands et moyens mammifères fréquentant les clairières (ou Baï) forestières de la zone de Pondo située dans sa partie Sud. Méthodologie et résultats : Elle a été conduite du 20 Avril au 20 Septembre 2015. La méthode de Recces et les observations directes effectuées entre 07h-12h et 14h$17 \mathrm{~h} 30$ ont été appliquées. Les fiches de collecte ont été utilisées pour recueillir les informations. Un total de 20 espèces de grands et moyens mammifères a été recensé dont 06 observés dans tous les sites : cercocèbe à joues grises (Cercocebus albigena), céphalophe de Peters (Céphalophe de Peters), éléphant de forêt (Loxodonta Africana cyclotis), hocheur (Cercopithecus nictitans), gorille (Gorilla gorilla), potamochère (Potamochoerus porcus). La saline a la plus grande richesse spécifique avec $90 \%$ d'espèces et le site le plus fréquenté. Le braconnage est l'activité anthropique dominante avec un taux de rencontre de 9 indices au $\mathrm{km}$.

Conclusion et application : II ressort de cette étude que le complexe de Baïs de Pondo est une zone de forte concentration animale en particulier pour les primates, les éléphants de forêt et les céphalophes qui y trouvent des conditions idéales pour leur survie et épanouissement. Malheureusement, ces espèces font face à plusieurs menaces entre autres le braconnage qui est l'activité anthropique dominante. Par conséquent, la sécurisation de cette zone est primordiale afin d'assurer la préservation/conservation de ses espèces. Notamment à travers des campagnes de sensibilisation des populations riveraines et l'amélioration de leurs conditions de vie, campagnes régulières de lutte anti-braconnage et le renforcement des capacités du service de conservation du parc national de Boumba Bek.

Mots clés : Grands et moyens mammifères, Clairières forestières ; Suivi écologique ; Parc National de Boumba-Bek. 


\title{
Ecological monitoring of the dynamics of large and medium mammals in the clearings of Boumba Bek National Park: Case of the Pondo clearing complex
}

\begin{abstract}
Objectives: The study in Boumba Bek National Park aimed to determine the status of the large and medium-sized mammal animal population frequenting the forest clearings of the southern part of Pondo area.

Methodology and results: The study conducted from April 20 to September 20, 2015The Recces method and the direct observations made between 07:00-14:00 p.m and 14:00-17:30 pm were applied. The data were recorded in collection sheets. A total of 20 species of large and medium mammals were recorded, out of which 6: grey-cheeked mangabey (Cercocebus albigena), Peters's duiker (Cephalophos of Peters), African forest elephant (Loxodonta Africana cyclotis), greater Spot-nosed monkey (Cercopithecus nictitans), gorilla (Gorilla gorilla), and red river hog (Potamochoerus porcus) were observed in all the sites. Clearings had the greatest specific richness with $90 \%$ species and the most frequented site. Poaching is the dominant anthropogenic activity with a meeting rate of 9 indices per $\mathrm{km}$.

Conclusion and application: This study revealed that Pondo Baïs complex is an area highly populated with animals especially primates, elephants, and duiker that find ideal conditions for their survival and development. However, these species face a number of threats such as poaching, which is the dominant human activity. Therefore, securing this area is necessary if these animal species are to be conserved. Campaigns to raise awareness among residents, anti-poaching campaigns and capacity building of the conservation service of Boumba Bek National Park are powerful tools to conserve the Parks and improve the living standards of the community through tourism generated revenue.
\end{abstract}

Keywords: Large and medium mammals, Forest clearings; Ecological monitoring; Boumba-Bek National Park.

\section{INTRODUCTION}

La création de périmètre de conservation du milieu naturel (faune, flore, sol, sous-sol, atmosphère, eaux) présente de nos jours un intérêt particulier notamment pour la préservation des milieux forestiers contre tout effort de dégradation. C'est ainsi que le Cameroun afin de protéger son patrimoine forestier et ses écosystèmes a créé plusieurs aires protégées parmi lesquelles le parc national de Boumba Bek (PNBB) par décret $\mathrm{N}^{\circ} 2005 / 3284 / \mathrm{PM}$ du 06 octobre 2005 (MINFOF, 2011). Ce parc, représente à lui seul plus de 37 espèces de grands mammifères dont 11 espèces de primates, 12 espèces d'ongulés et 04 espèces de carnivores (MINFOF, 2011 ; Nzooh et al., 2016). Malheureusement cette zone de conservation qui s'étend sur les arrondissements de Moloundou, Salapoumbé et Yokadouma fait face à plusieurs menaces qui pourraient en l'absence de prises de décisions de gestion adéquate en altérer l'aspect ou la composition (Ngaba et al., 2019). II est dès lors primordial de collecter des informations de façon régulière sur sa biodiversité, le milieu, dans l'espace et le temps et, en vue de mieux le connaître et déceler les changements éventuels, pour une prise de décision de gestion. De plus, La connaissance du potentiel faunique d'une aire protégée est un outil indispensable pour son aménagement et sa gestion durable. Pour se faire, les gestionnaires des parcs utilisent le suivi écologique. Le PNBB regorge par ailleurs des habitats ouverts communément appelé "bais" ou clairières qui sont régulièrement fréquentées par les animaux tels que les éléphants de forêt (Loxodonta africana cyclotis), les buffles de forêt (Syncerus caffer nanus), les gorilles de plaine (Gorilla gorilla), les potamochères (Potamochoerus porcus) qui y vont en vue de s'abreuver, se nourrir ou se divertir. D'après Magliocca et al. (2011), la disponibilité d'une végétation et d'eau très riche en sels minéraux (sodium, calcium, magnésium, etc.) qu'on retrouve dans ces milieux tout au long de 
l'année constitue pour ces animaux une source de complément alimentaire. La zone de Pondo située dans sa partie Sud est constituée de plusieurs clairières forestières qui offrent des opportunités d'observations par excellence de la faune sauvage dans le cadre des activités du suivi écologique, outil indispensable pour son aménagement et sa gestion durable. Cette zone a déjà fait l'objet au paravent d'une étude (Ngaba, 2015) qui a révélé la présence d'une forte diversité faunique, d'un fort taux de fréquentation de ses clairières et que cette zone peut par conséquent faire l'objet d'une valorisation écotouristique. Toutefois, il a été observé lors de cette étude un comportement

\section{MATERIELS ET METHODES}

Localisation de la zone d'étude: L'étude a été réalisée dans le complexe des clairières forestières de Pondo situé entre $2^{\circ} 45^{\prime}$ et $2^{\circ} 48^{\prime}$ latitude Nord et entre $14^{\circ} 98^{\prime}$ et $15^{\circ} 01^{\prime}$ longitude Est dans la partie Sud du PNBB à près de $1500 \mathrm{~m}$ de la rivière Bek, affluent de la grande rivière Boumba (Figure 1). Le climat est de type équatorial guinéen, fortement marqué par la continentalité avec 04 saisons et les précipitations s'étalent tout au long de l'année avec deux pics en avril diurne des certaines espèces emblématiques dont l'éléphant de forêt, ce qui est une menace non négligeable à la mise en place d'une activité écotouristique (tourisme de vision). De plus, cette étude n'a pas pu confirmer à travers des observations directes leurs présences, ce qui peut s'expliquer à travers la méthodologie utilisée. La présente étude s'est ainsi donnée pour objectif : (1) D'étudier le statut des animaux (richesse spécifique, abondance relative, fréquence) ; (2) De faire une comparaison de la diversité d'espèces observées entre les sites d'étude ; (3) D'identifier les pressions anthropiques rencontrées dans la zone et sa périphérie.

et en octobre (Harrison et Agland, 1987 ; Ekobo, 1995). La température annuelle moyenne est de $24{ }^{\circ} \mathrm{C}$ et l'humidité relative de l'air varie de 60 à $90 \%$. Les sols de la zone appartiennent au sous-ordre des sols ferralitiques typiques définis par une séparation du fer et de l'oxyde d'alumine, sont de type argileux avec une fine couche d'humus superficiels contenant peu de matière organique. Ils sont acides et pauvres en azote et bases échangeables (Noupa et al., 2008).

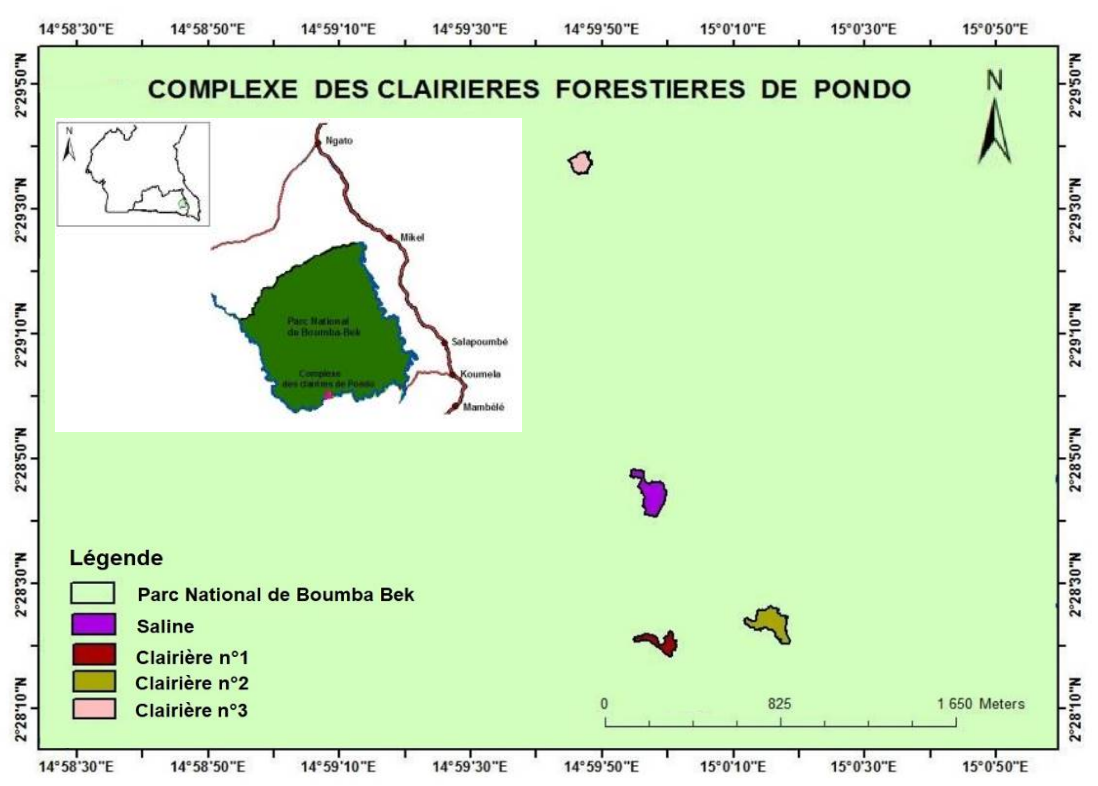

Figure 1: Localisation du complexe de Pondo dans le PNBB 
Caractérisation des clairières du complexe de Pondo: Les clairières $n^{\circ} 1,2$ et 3 ont des sols hydromorphes, ce sont des marécages périodiquement inondés, colonisés par un tapis herbacé (Photo 1A). La végétation de ces clairières est composite, constituée de Graminées, d'Astéracées, de Filicinées, de Cypéracées, de Comelinacées, de Raphiales et Marantacées ; espèces caractéristiques des marécages à l'intérieur des clairières (Belinga, 2015). La végétation des lisières quant à elle est constituée d'arbres tels que Mellettia mannii, Maytenus sp., Hallea stipulosa. La saline $n^{\circ} 1$ est périodiquement traversée par un petit cours d'eau surtout en saison de pluie dans sa périphérie. Elle est couverte par endroit par une végétation herbacée dans la site et arbuste au niveau des périphéries (Photo 1B).
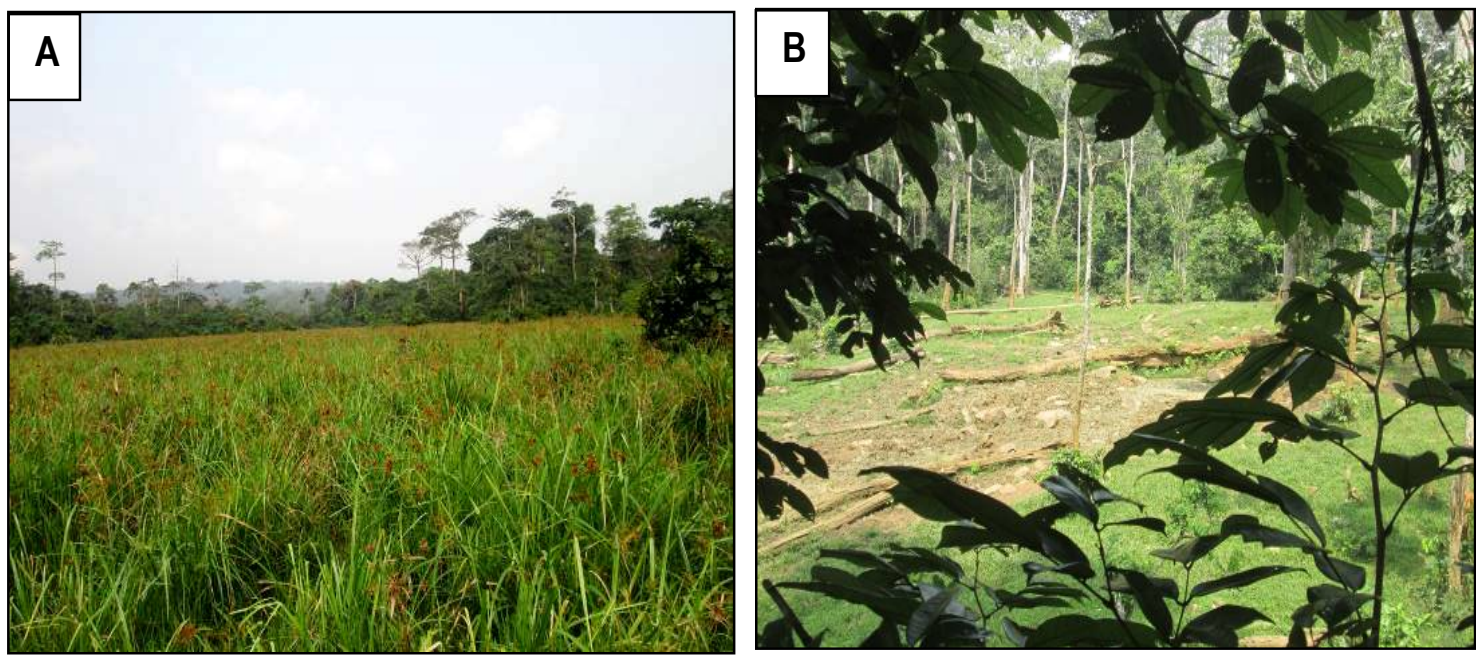

Photo 1: Sites d'observation. Clairière forestière $n^{\circ} 1(A)$ et saline $(B)$

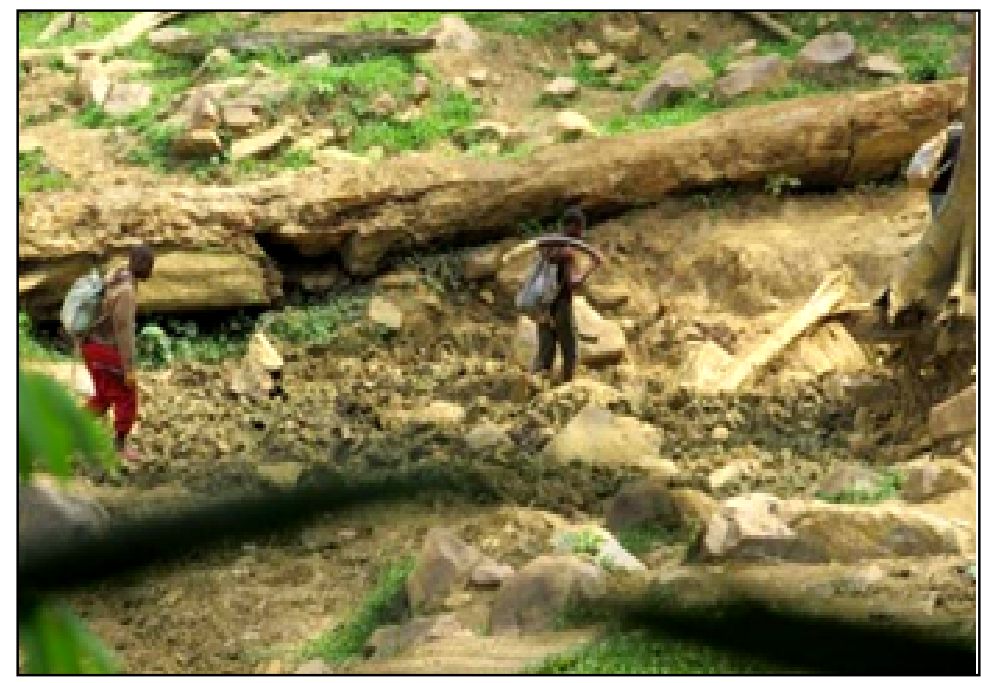

Photo 2: Braconniers en déplacement observés dans la saline.

Méthodologie : La collecte des données s'est déroulée du 20 Avril au 20 Septembre 2015. Chaque décente avec une durée de 10 jours et l'intervalle de temps entre les décentes étaient de 10 jours. Une équipe de 05 personnes a été mobilisé : 02 guides locaux pour l'identification des animaux et leurs signes ; 02 écogardes afin d'assurer la sécurité et ayant une bonne connaissance de la zone d'étude et 01 ingénieur des eaux et forêt pour la collecte des informations.

Les observations directes. Elles ont été effectuées dans les sites entre $07 \mathrm{~h}-12 \mathrm{~h}$ et $14 \mathrm{~h}-17 \mathrm{~h} 30$. Les observations étaient faites sur des perchoirs à l'aide de jumelles et d'un appareil photo afin de mieux visualiser les animaux. Les informations collectées, le type d'espèces, leurs activités, le sexe, la structure d'âge et 
taille des groupes d'espèces. Trois classes d'âges ont été considérées: adulte, sub-dulte et juvénile en se basant sur le dimorphisme sexuel. Au cas où un individu n'a pas pu être classé dans l'une des trois catégories, il était systématiquement classé comme "Indéterminé".

Les observations indirectes. La méthode de « Recces» a été appliquée (Maisels et al., 2005). Les indices recherchés étaient les indices de présence des animaux (crottes, empreintes, traces d'alimentation, bauges, ) et anthropiques (empreintes de pas, douilles de cartouches, pièges, campements ). Leurs états étaient aussi collectés (frais, récents, vieux et fossilisé). Chaque observation était collectée sur des fiches et géoréférencées.

Analyses statistiques: Le taux de rencontre moyen ou IKA moyen (indice kilométrique d'abondance moyen). Les indices kilométriques d'abondance (Bousquet, 1996) nous ont permis d'estimer l'abondance relative des populations animales et des activités anthropiques de la zone d'étude. Elles ont été calculées avec la formule suivante :

\section{IKA $=\underline{\text { Nombre de contact avec une espèce }}$ Distance totale parcourue}

L'indice de diversité de Shannon-Weaver $\left(H^{\prime}\right)$. II a servi à mesurer ou évaluer le niveau d'organisation des types d'habitats. La diversité est perçue dans les systèmes étudiés comme une mesure de l'organisation du système et du degré de sensibilité aux

perturbations. Une organisation optimale sera celle pour laquelle les perturbations ne pourront avoir qu'un impact réduit sur la biodiversité (Shannon et Weaver, 1963). La formule suivante a été utilisée :

$$
\mathrm{H}^{\prime}=\sum\left[\left(n_{i} / \mathrm{N}\right) \log 2\left(n_{i} / \mathrm{N}\right)\right]
$$

Où ni : Nombre d'individus d'une espèce i, pour i allant de 1 à $S$ (nombre total d'espèces); $N$ : Nombre total d'individus de toutes les espèces.

La fréquence d'observation directe

$$
\mathrm{F}=\frac{\mathrm{N}_{i}}{\mathrm{~N}_{j}}
$$

Où $\mathrm{Ni}$ : Nombre d'observations d'une espèce durant une période de temps i ; $\mathrm{j}$ : Nombre de jours durant laquelle les observations ont été faites.

\section{RESULTATS ET DISCUSSION}

Richesse spécifique: Un total de 20 espèces de grands et moyens mammifères réparties dans 04 ordres, 09 familles et 03 classes de protection existantes au Cameroun dont 09 espèces de classes A, 06 espèces de classe $B$ et 05 espèces de classe $C$. Ces résultats différents de ceux obtenus par Bene \& Nzooh (2005) avec 25 espèces répertoriées et Ngaba (2014) avec 16 espèces répertoriées. Cette différence peut être due à la taille du site d'étude et la méthodologie utilisée. En effet, l'étude réalisée par Bene Bene \& Nzooh (2005) avait couvert tout le PNBB lors des inventaires fauniques. En outre, il en ressort que 06 espèces été recensées dans tous les sites: cercocèbe à joues grises (Cercocebus albigena), céphalophe de Peters (Céphalophe de Peters), éléphant de forêt (Loxodonta Africana cyclotis), hocheur (Cercopithecus nictitans), gorille (Gorilla gorilla), potamochère (Potamochoerus porcus) et que la saline a la plus grande richesse spécifique avec $90 \%$ d'espèces. Cette inégale répartition des espèces dans toute la zone d'étude peut s'expliquer à travers les caractéristiques des différents sites. En effet, la saline se démarque premièrement des autres sites par son accessibilité car le site est tapissé par une végétation herbacée par endroits et n'est pas inondée. De plus, son sol riche en sels minéraux et fraiches repousses d'herbes ce qui attire encore plus d'animaux. Par ailleurs, les espèces telles que le Chevrotin aquatique 
et le Sitatunga semblent avoir une préférence à vivre dans les milieux humides (inondés ou périodiquement inondés). Ces observations stipulent que les animaux s'orientent vers des milieux qui sont favorables à leur épanouissement.

Indices de présence et observation des animaux entre les sites: D'une manière générale, 462 signes d'activités ont été observés pendant l'étude dans les différents sites sur une distance totale de $4,3 \mathrm{~km}$, avec $15,15 \%$ d'observations directes et $84,85 \%$ d'observations indirectes. La saline a le plus grand nombre de signes d'activités observés indirectement $(73,21 \%)$ et directement $(84 \%)$. Les analyses statistiques nous montrent qu'il y a une différence significative $(P<0,01)$ entre la saline et les clairières. Toutefois, aucune différence significative n'a été observée entre les clairières $(P=0,07)$.

L'abondance relative des animaux tel que : Le hocheur, le cercocèbe à joues grises, le cercopithèque de Brazza, le buffle nain et le gorille montrent une abondance relativement très élevée avec respectivement 25,$1 ; 15,6 ; 8,8 ; 7,9$ et 7,2 indices au kilomètre. Les espèces les plus faiblement représentées sont le Talapoin, Chevrotain aquatique et le Sitatunga avec respectivement 0,$2 ; 0,7$ et 0,7 signe au kilomètre.

Période journalière de visite, durée de fréquentation des animaux: Les résultats de cette étude ont démontré que les animaux fréquentent la zone d'étude dans la matinée $(69,5 \%)$ que dans l'après-midi $(30,5 \%)$ sans doute parce qu'ils sont moins exposés aux rayons de soleil. En effet, lors des marches de reconnaissance entre $11 \mathrm{~h}$ et $12 \mathrm{~h} 30 \mathrm{~min}, 87 \%$ des animaux qui ont été observé étaient sous un arbre. Similaires aux résultats obtenus dans d'autres études (Abou, 2014 ; Ngaba, 2014). II découle des analyses que les sites sont plus fréquentés entre $10 \mathrm{~h}$ et $11 \mathrm{~h}$. La durée des animaux dans les sites variait de 01 à $80 \pm 7$ minutes. Les animaux qui mettaient le plus de temps dans les sites sont les cercocèbes à joues grises (80 minutes), suivi des hocheurs et pogonias (67 minutes) et des gorilles (45 minutes).

Comportements des animaux et structure d'âge des animaux : Les activités des animaux ont été recensées dans la Figure 2. Les animaux avaient un comportement " craintif ». Deux hypothèses peuvent expliquer cette attitude. La première est liée à un facteur naturel : l'instinct de survie des animaux lorsqu'ils font face à toutes menaces potentielles. $\mathrm{Ce}$ réflexe de fuite est accentué par la présence de félins (panthères) dans la zone. La deuxième est le braconnage qui sévit dans la zone à travers le stress lié au coup de feu... Ces deux hypothèses sont les principaux facteurs qui augmentent la distance de fuite des animaux (6 à $13 \mathrm{~m}$ ).

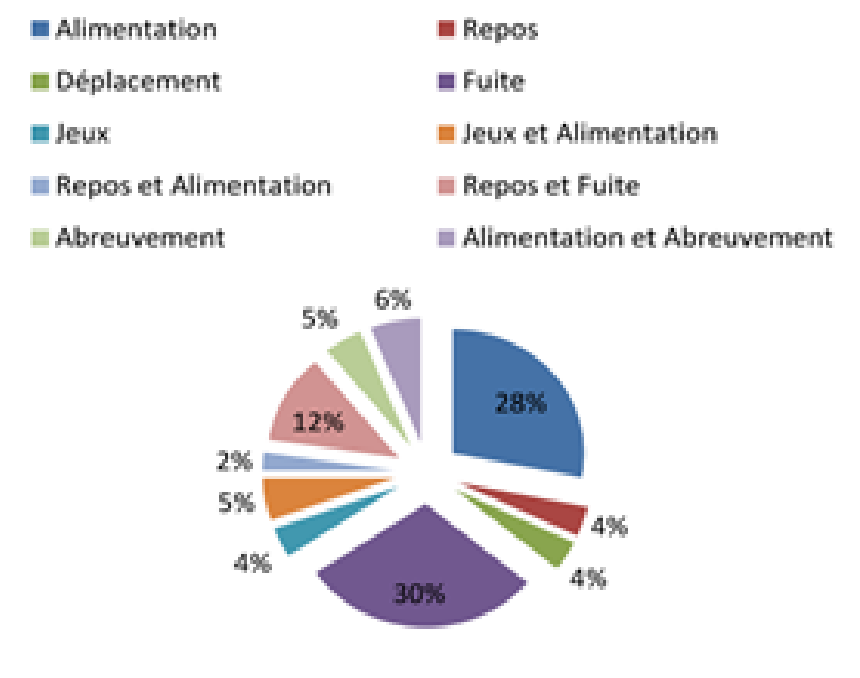

Figure 2: Répartition des comportements/activités des animaux dans les sites. 
Fréquence d'observation des indices d'activités dans les sites

Observations directes des indices d'activités dans

les sites: Trois groupes d'espèces ont été classés à cet effet : les espèces régulières, les espèces non régulières et les espèces rares. Les espèces observées de façon régulières : l'hocheur $(28,6 \%)$, le cercocèbe à joues grises $(24,3 \%)$ et le céphalophe de Peters $(17 \%)$ ; Les espèces observées de manière intermittente : Parmi elles nous pouvons citer le Cercocèbe agile $(7,1 \%)$ et le Gorille $(5,7 \%)$; Les espèces rares ou difficilement observables : la panthère, le potamochère, le sitatunga, le cercopithèque de Brazza et le céphalophe à dos jaune. Ces résultats diffèrent de ceux obtenus par Abou (2014) dans les clairières de Batouka, Likolo et Djewa où le Buffle nain est l'espèce dont les observations directes ont été plus régulières. Les éléphants de forêt, les Buffles nains et les Bongos semblent visiter les clairières dans la nuit et très tôt le matin plutôt que dans la journée car des indices frais de ceux-ci ont été retrouvés très tôt le matin dans les sites. Cette observation confirme les résultats des études menées à Pondo par Ngaba (2014) et Belinga (2015). Deux hypothèses peuvent expliquer ce comportement. La première est liée à un facteur naturel lié à l'instinct de survie des animaux sans doute due à la présence de carnivores féroces (panthère) dans la zone d'étude. La seconde est liée au braconnage, confirmé par les indices d'activités anthropiques observés (coups de feu, empreintes de pas, coupes de machette).

Observation des indices indirects des animaux dans les sites: Les espèces ayant plus d'indices indirects de présence ou dont les signes indirects ont été observés de manière régulière ; elles sont au nombre de 02 : l'hocheur $(22,4 \%)$ et le cercocèbe à joues grises $(12,8 \%)$; Les espèces non régulières dont les indices indirects de présence ont été relevés de manière intermittente. Parmi elles nous pouvons citer le cercopithèque de Brazza $(9,7 \%)$, le gorille $(6,9 \%)$ et le buffle nain $(8,2 \%)$; les espèces rares ou difficilement observables : elles sont au nombre de 06 : la panthère $(1,8 \%)$, le potamochère $(1,8 \%)$, le cercocèbe agile $(1,8 \%)$, le sitatunga $(0,8 \%)$, le chevrotain aquatique $(0,8 \%)$ et le talapoin $(0 \%)$ (Tableau 1$)$. Le faible taux

\section{REMERCIEMENTS}

Cette étude a été coordonnée sous le projet JengiTRIDOM (WWF/Cameroun) à qui nous témoignons notre reconnaissance. Nous exprimons aussi notre gratitude au Chef de Département de Foresterie de la d'indices répertoriées dans les clairières peut s'expliquer par la présence d'eau qui inonde ses milieux et rendent difficile l'observation des signes d'activités des animaux. Par ailleurs, la probabilité d'observer les animaux et sur une longue durée était plus élevée dans la saline que dans les clairières.

Etat des lieux des activités anthropiques dans le site d'étude et sa périphérie: Le braconnage se présente comme l'activité la plus importante avec un taux de rencontre de 9 indices au $\mathrm{km}$. Plusieurs signes ont été rencontrés notamment les campements actifs ou abandonnés, les trophées abandonnés, les empreintes même des braconniers, les douilles de cartouches vides. Deux différents groupes de 03 et 07 braconniers armés ont été observé dans la saline. Un des groupes (constitué de 07 membres) transportaient 04 pointes d'ivoires d'Eléphants (Photo 2). Ceux-ci étaient munis de machettes, haches et armes de guerre (Kalashnikov). L'observation directe de ses individus nous a permis de déduire grâce à leurs traits physiques et la langue de communication utilisée que la plupart les braconniers viennent des villages riverains, ils ont été recrutés pour leur bonne connaissance du milieu et leur capacité à pister les animaux. Des résultats similaires ont été obtenu par Bene Bene \& Nzooh (2005) avec 0.8 indices au km et Ngaba (2014) avec 9,3 indices au $\mathrm{km}$. Bien que l'observation directe de braconniers et de carcasses confirment la menace et pression exercée sur la population de grands et moyens mammifères en général et sur la population d'éléphants en particulier, le coefficient de détermination $\left(R^{2}=0,4309\right)$ soit $43,09 \%$, démontre que le degré de liaison entre les activités anthropiques et la dynamique des populations animales dans les sites n'est pas significatif. Toutefois, l'analyse des données (droite de régression : $y=-0,019+4,357$ ) nous prouve que les activités anthropiques ont une influence négative sur la dynamique des populations de grands et moyens mammifères dans la zone de Pondo. Le coefficient de corrélation de Spearman montre que l'intensité de la liaison entre les IKA de la faune des sites d'étude et les IKA des activités anthropiques est négative a pour valeur $r=-0,73$.

Faculté d'Agronomie et des Sciences Agricoles (FASA) de l'Université de Dschang, au Coordonnateur du Chef de programme Jengi-TRIDOM et son équipe, aux Services de conservation du PNBB et PNK. 


\section{BIBLIOGRAPHIE}

Abou, 2014. Suivi écologique des grands et moyens mammifères dans certaines clairières du Parc National de Boumba-Bek, mémoire de fin d'étude FASA de Université de Dschang.

Belinga BJP, 2015. Rapport de suivi écologique de la dynamique de la faune sauvage dans le complexe des clairières de Pondo au Parc National de Boumba-Bek, WWF, 2015.

Bene BCL, Nzooh ZL, Usongo L, 2002. Résultats préliminaires du suivi écologique dans le Parc National de Boumba Bek. Rapport WWF CARPO. 25pp. Bousquet, 1996. Nature Reserve management in China. GEF/Project, the World Bank.

Ekobo A, 1995. Conservation of the African forest elephant (Loxodonta africana cyclotis) in Lobeke, Southeast Cameroon. PhD thesis, 151 pp Harrison et Agland, 1987

Harrisson M, Agland P, 1987. A draft proposal for the designation of three new national forest parks. Dja river Films, LTD.

Magliocca F, Querouil S, Gautier-hion A, 1999. Population structure and group composition of Western lowland gorillas in north-western Republic of Congo. American Journal of Primatology, 48(1): 1-14.

Maisels F, 2005. Ivindo monitoring brochuretransects12/17/2005. Wildlife Conservation Society (Rapport interne).
MINFOF (Ministère des Forêts et de la Faune), 2011. Plan d'aménagement de Boumba-Bek

Ngaba MJY, 2015. Suivi écologique de la dynamique des grands et moyens mammifères dans les clairières du parc national de Boumba-Bek: Cas du complexe de Pondo. Doi : 10.13140/RG.2.2.36383.61600.

Ngaba MJY, 2019. Suivi écologique de la dynamique des grands et moyens mammifères dans les clairières du parc national de Boumba-Bek : Cas du complexe de Pondo.

Noupa P, Nkongmeneck BA, 2008. Influence des clairières forestières sur la répartition spatiale des grands mammifères dans la forêt dense du Bassin du Congo : cas du Parc National de Boumba-Bek (Sud-est Cameroun). International Journal of Biological and Chemical Sciences, 2(2): p, 185-195. Doi: 10.4314/ijbcs.v2i2.39734.

Nzooh ZL, N'goran, Etoga G, Belinga JP, 2016. Les populations de grands et moyens mammifères dans le segment Cameroun du paysage TRIDOM (forets de Ngoyla MINTOM, PN Boumba-Bek, PN Nki et leurs périphériques).

Shannon CE and Weaver W, 1963. The mathematical theory of communication. Champaign, IL : University of Illinois Press. Sutherland, 2001.

Tableau 1 : Abondance relative et signes de présence animale par espèces d'Avril à Septembre 2015.

\begin{tabular}{|l|c|c|c|c|c|}
\hline \multicolumn{1}{|c|}{ Noms communs et scientifiques } & $\begin{array}{c}\text { observations } \\
\text { directes }\end{array}$ & $\begin{array}{c}\text { Indices } \\
\text { indirects }\end{array}$ & Total & $\begin{array}{c}\text { Longueur des } \\
\text { recces }\end{array}$ & IKA \\
\hline Bongo (Bocerus eurycerus) & 1 & 11 & 12 & 4,3 & 2,8 \\
\hline $\begin{array}{l}\text { Buffle nain } \\
\text { (Syncerus caffer nanus) }\end{array}$ & 2 & 32 & 34 & 4,3 & 7,9 \\
\hline $\begin{array}{l}\text { Cercopithèque de brazza } \\
\text { (Cercopithecus neglectus) }\end{array}$ & 0 & 38 & 38 & 4,3 & 8,8 \\
\hline $\begin{array}{l}\text { Cercocèbe à joues grises (Cercocebus } \\
\text { albigena) }\end{array}$ & 17 & 50 & 67 & 4,3 & 15,6 \\
\hline $\begin{array}{l}\text { Cercocèbe agile } \\
\text { (Cercocebus Agilis) }\end{array}$ & 5 & 7 & 12 & 4,3 & 2,8 \\
\hline $\begin{array}{l}\text { Céphalophe à bande dorsale noire } \\
\text { (Cephalophus dorsalis) }\end{array}$ & 1 & 11 & 12 & 4,3 & 2,8 \\
\hline $\begin{array}{l}\text { Céphalophe à dos jaune (Cephalophus } \\
\text { silvicultor) }\end{array}$ & 0 & 9 & 9 & 4,3 & 2,1 \\
\hline $\begin{array}{l}\text { Céphalophe de peters } \\
\text { (Cephalopus callipygus) }\end{array}$ & 12 & 12 & 24 & 4,3 & 5,6 \\
\hline
\end{tabular}


Imbey et al., J. Appl. Biosci. 2019 Suivi écologique de la dynamique des grands et moyens mammifères dans les clairières du parc national de Boumba Bek : cas du complexe de clairières de Pondo

\begin{tabular}{|l|c|c|c|c|c|}
\hline $\begin{array}{l}\text { Céphalophe bleu } \\
\text { (Cephalophus monticola) }\end{array}$ & 2 & 21 & 23 & 4,3 & 5,3 \\
\hline $\begin{array}{l}\text { Chevrotain aquatique } \\
\text { (Hyemoschus aquaticus) }\end{array}$ & 0 & 3 & 3 & 4,3 & 0,7 \\
\hline Chimpanzé (Pan troglodytes) & 1 & 11 & 12 & 4,3 & 2,8 \\
\hline Colobe guéréza (Colobus guereza) & 1 & 25 & 26 & 4,3 & 6 \\
\hline $\begin{array}{l}\text { Éléphant de forêt } \\
\text { (Loxodonta Africana cyclotis) }\end{array}$ & 1 & 9 & 10 & 4,3 & 2,3 \\
\hline Gorille (Gorilla) & 4 & 27 & 31 & 4,3 & 7,2 \\
\hline $\begin{array}{l}\text { Hocheur } \\
\text { (Cercopithecus nictitans) }\end{array}$ & 20 & 88 & 108 & 4,3 & 25,1 \\
\hline Pogonias (Cercopithecus) & 2 & 21 & 23 & 4,3 & 5,3 \\
\hline Panthère (Panthera pardus) & 0 & 7 & 7 & 4,3 & 1,6 \\
\hline $\begin{array}{l}\text { Potamochère } \\
\text { (Potamochoerus porcus) }\end{array}$ & 0 & 7 & 7 & 4,3 & 1,6 \\
\hline $\begin{array}{l}\text { Sitatunga } \\
\text { (Tragelaphus spekii) }\end{array}$ & 0 & 3 & 3 & 4,3 & 0,7 \\
\hline Talapoin (Miopithecus talapoin) & 1 & 0 & 1 & 4,3 & 0,2 \\
\hline Total & 70 & 392 & 462 & $\mathbf{4 , 3}$ & $\mathbf{1 0 7 , 2}$ \\
\hline
\end{tabular}

